\title{
Evaluation of effect of adaptive motion on shaping ability of twisted file nickel-titanium files in simulated S-shaped canals
}

\author{
Taha Özyürek, Ebru Özsezer Demiryürek \\ Department of Endodontics, Ondokuz Mayıs University Faculty of Dentistry, Samsun, Turkey
}

\begin{abstract}
Objective: To evaluate the effect of adaptive motion on shaping ability of Twisted Files nickel-titanium files in simulated S-shaped canals.

Methods: Forty S-shaped canals in resin blocks were prepared to an apical size of $0.25 \mathrm{~mm}$ using Twisted Files with rotary or adaptive motion ( $n=20$ canals/group). Composite images were made from the superimposition of pre- and post-instrumentation images. The amount of resin removed by each system was measured by using a digital template and image analysis software. Canal aberrations were also recorded. The data were statistically analyzed by using Mann-Whitney $\mathrm{U}$ test.
\end{abstract}

Results: There was no significantly difference between adaptive and rotary motion groups $(P>0.05)$. No instrument fracture or canal aberrations were inspected during canal preparation.

Conclusion: Within the limitation of the present study, adaptive and rotary motions were safe to use and were able to prepare the canals efficiently.

Keywords: Adaptive motion; endodontics; nickel-titanium; twisted file.

$\mathrm{S}^{\mathrm{s}}$

haping the root canals gradually increasing from apical to coronal is one of the most important steps for sufficiently cleaning and filling the root canals. ${ }^{[1]}$ The nickeltitanium (NiTi) alloys having super flexibility are useful in minimizing the canal irregularities such as ledge, zip and perforations that may occur during shaping the narrow and curved canals. ${ }^{[2,3]}$ Besides these advantages of the NiTi alloys, they have an important disadvantage that they have high fracture incidence due to the cyclic fatigue in the narrow and curved canals. Nowadays, the NiTi rotary file systems (Conventional, R-Phase, M-Wire, and Gold-Wire $\mathrm{NiTi}$ ) made of various alloys and with various kinematics (continuous rotation, reciprocation, and adaptive motion) are recommended for maintaining the original canal form during shaping the root canals..$^{[4,5]}$

Twisted File Adaptive (TFA; SybronEndo, Orange, (A, USA) is a system that operates with its specific endodontic motor (Elements Motor; SybronEndo) and aiming to combine the advantages of rotation (continuous $360^{\circ}$ clockwise motion) and reciprocation (clockwise and counterclockwise motion at different degrees). In case that there is no stress in the canal, the file completes its $600^{\circ}$ clockwise rotation, then stops and continues rotational motion. As the stress on file increases, Elements Motor modifies the motion up to $370^{\circ}$ clockwise and $50^{\circ}$ clockwise motion depending on the level of stress. ${ }^{[6]}$ As a result of kinematic modification, the stress on file decreases through the counterclockwise motion of file, when

Correspondence: Dr. Taha Özyürek. Ondokuz Mayıs Üniversitesi, Diş Hekimliği Fakültesi,

Endodonti Anabilim Dalı, 55137 Samsun, Turkey.

Tel: +90 362 - 3121919 e-mail: tahaozyurek@hotmail.com

Submitted: December 26, 2016 Accepted: January 20, 2017

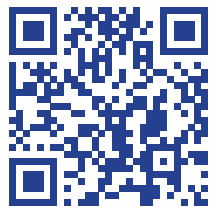


compared to continuous rotation motion.

In comprehensive search of the literature, it was determined that there is limited number of studies on the effects of adaptives motion on shaping ability of Twisted File (TF) files. ${ }^{[7,8]}$ Pedullà et al. ${ }^{[7]}$ analyzed the effects of adaptive motion on the efficiency of TF NiTi files in shaping the mandibular molar teeth having $25^{\circ}-35^{\circ}$ of curvature by using computed tomography. In literature, there is no study examining the effects of adaptive motion on shaping S-shaped artificial canals. For these reasons, the aim of present in vitro study was to examine the effects of adaptive motion on the shaping ability of Twisted File NiTi rotary file system in S-shaped canals. The null hypothesis of present study was that continuous rotation motion and adaptive motion would have no effect on the shaping ability of Twisted File.

\section{Materials and methods}

Forty S-shaped artificial canals (Endo Training Block-S; Dentsply Maillefer, Ballaigues, Switzerland) having 0.02 taper and $0.15 \mathrm{~mm}$ of apical diameter with $16 \mathrm{~mm}$ of working length were used in the present study. Coronal angle of curvature of artificial canals was $30^{\circ}$ and radii of curvature was $5 \mathrm{~mm}$; the apical angle of curvature was $20^{\circ}$, and radii of curvature was $4.5 \mathrm{~mm}$. The artificial canal openings were checked using \#10 K-file (Dentsply Maillefer), and then the specimens were randomly divided into 2 groups $(n=20 /$ each). Then the following procedures were implemented:

\section{Group 1: Twisted file}

In accordance with crown-down method, the canals in this group were prepared by using TF 25/.06 NiTi file with torque-controlled endodontic motor (Elements Motor) at $500 \mathrm{rpm}$ and $3 \mathrm{Ncm}$ torque.

\section{Group 2: Twisted file adaptive}

In accordance with crown-down method, the canals in this group were shaped using TFA SM2 (25/.06) NiTi file with torque-controlled endodontic motor (Elements Motor) via "TF Adaptive" program.

All of the procedures were performed by an endodontist, who was experienced in the use of TF NiTi files. Each of the canals was prepared using a new NiTi file. During the use of files, the canals were irrigated using a total of $20 \mathrm{~mL}$ distilled water. In total, 40 S-shaped artificial canals were shaped.

\section{Assessment of canal preparation}

Prior to the beginning of canal preparation procedure, all of the canals were stained using black ink (Pelikan, İstanbul, Turkey). The artificial canals were placed on a setup, where the canals can be placed always (before and after the shaping) in the same position. The photos of canals were taken using a camera (Canon EOS 500D, Japan) mounted on the same setup. After performing the shaping procedure, the canals were stained with red ink (Pelikan), and their photos were taken again in the same setup.

The photos of the canals before and after the procedure were superimposed using a computer program (Pages; Apple Inc., Cupertino, CA, USA), and the composite images were obtained (Figure 1). Using the same program, the measurement template, which was prepared for the measurements, was placed on the composite images. Then composite images were transferred to AutoCAD (Autodesk, San Rafael, CA, USA) and the amount of removed resins was measured at 22 points (11 internal and 11 external points). The measurement points were determined to have $1 \mathrm{~mm}$ increment; $0-3$ points were determined to be apical, 4-7 points to be middle, and 8-10 points to be coronal portion. ${ }^{[9]}$ Moreover, after the preparation of artificial canals, the width was measured at 11 points. Using the obtained data, the transportation caused by the tested files was calculated via the formula specified below.

Amount of transportation (mm): Amount of resin removed from inside the artificial canal ( $\mathrm{mm}$ ) - Amount of resin removed from outside the artificial canal $(\mathrm{mm})$.

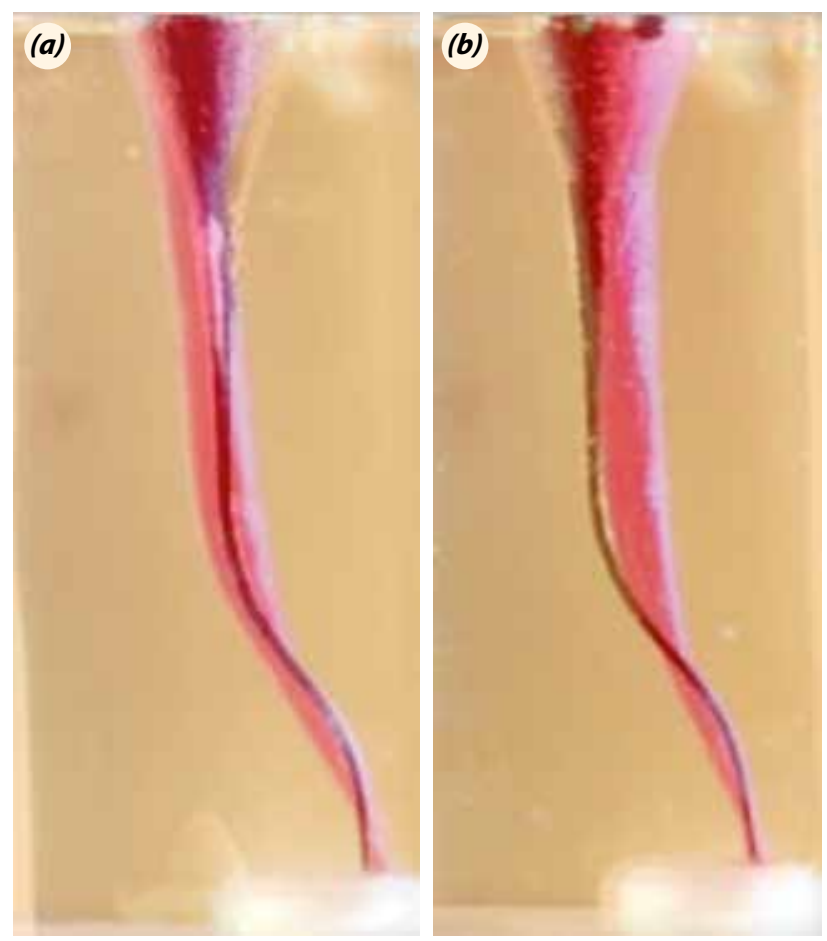

Fig. 1. The composite images of the simulated S-shaped canals ([a] TFA; [b] TF). 


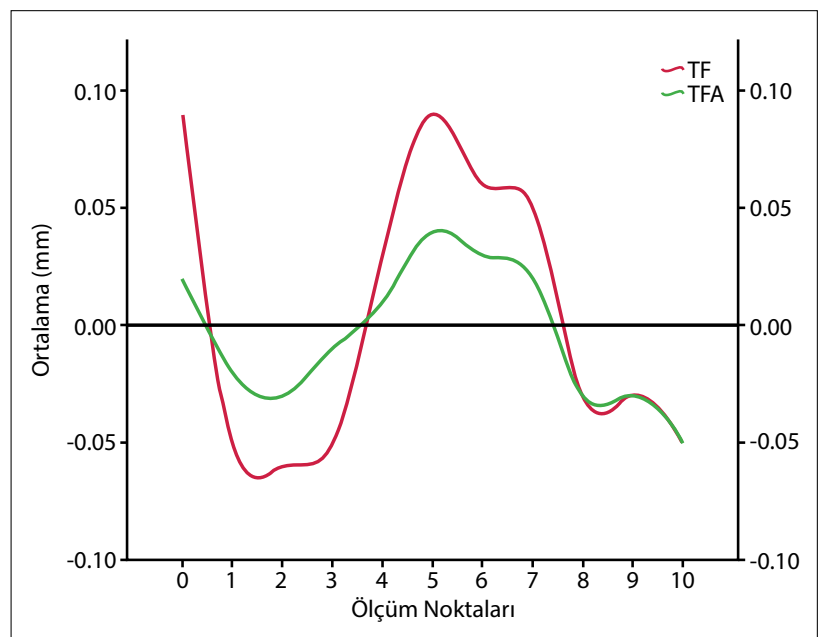

Fig. 2. The transportation values of the tested NiTi files in the measuring points $(\mathrm{mm})$.

\section{Statistical analysis}

The analysis of obtained data was performed using SPSS 21 (IBM-SPSS Inc., Chicago, IL, USA) program. The normality of the data was verified for each set of measurements by using the Shapiro-Wilk test. The difference between the groups was examined using Mann-Whitney $U$ test. Statistical significance was set at $5 \%$.

\section{Results}

No canal irregularity or NiTi file fracture was observed during preparation of the canals. From the aspect of mean amount of canal transportation made by the files, no statistically significant difference was found between the TF and TFA groups in apical (0-3 points), middle (4-7 points) and coronal (8-10 points) portion ( $p>0.05)$ (Figure 2).

Mean and standard deviation values of canal width

Table 1. The mean and standard deviations of simulated canal's width in the measurement points ( $\mathrm{mm}$ )

\begin{tabular}{ccc} 
& Twisted file & Twisted file adaptive \\
\hline 0 & $0.34 \pm 0.02$ & $0.32 \pm 0.02$ \\
1 & $0.39 \pm 0.04$ & $0.37 \pm 0.04$ \\
2 & $0.50 \pm 0.04$ & $0.48 \pm 0.02$ \\
3 & $0.55 \pm 0.02^{\mathrm{a}}$ & $0.51 \pm 0.02^{\mathrm{b}}$ \\
4 & $0.64 \pm 0.01^{\mathrm{a}}$ & $0.60 \pm 0.01^{\mathrm{b}}$ \\
5 & $0.73 \pm 0.02^{\mathrm{a}}$ & $0.69 \pm 0.02^{\mathrm{b}}$ \\
6 & $0.80 \pm 0.02^{\mathrm{a}}$ & $0.77 \pm 0.02^{\mathrm{b}}$ \\
7 & $0.80 \pm 0.02$ & $0.79 \pm 0.02$ \\
8 & $0.85 \pm 0.02$ & $0.84 \pm 0.02$ \\
9 & $0.89 \pm 0.02$ & $0.89 \pm 0.03$ \\
10 & $0.89 \pm 0.02$ & $0.89 \pm 0.03$ \\
\hline
\end{tabular}

*Different superscripts indicate statistically significant different $(p<0.05)$. shown at the measurement points on the canals shaped by TF and TFA file systems are presented in the table (Table 1). Statistically significantly lower amount of enlargement was determined between the 3-6 points of TFA group $(p<0.05)$.

\section{Discussion}

While gradually increasing enlarging the root canals from apical to coronal, it is important to maintain the original form of root canals. ${ }^{[1]}$ In many studies comparing the shaping abilities of NiTi files, S-shaped artificial canals were employed. ${ }^{[10-12]}$ In studies, where S-shaped canals were utilized, the shaping abilities of NiTi files can be easily compared via computer programs by superimposing the images of canals before and after the shaping procedure. ${ }^{[13]}$ The most important disadvantage of this technique is that the hardness value of dentin and resin, of which the artificial canals were made, are not the same, but determining the shaping characteristics becomes easier since the technique allows easily interpretation of the images before and after the procedure. ${ }^{[14]}$

In studies, where the NiTi files' shaping abilities were examined, different $\mathrm{NiTi}$ rotary file systems were analyzed using continuous rotation movement, reciprocation movement, and adaptive motion. ${ }^{[4,7,8,15]}$ But, only in 2 of these studies, the effect of adaptive motion on NiTi files shaping ability was examined..$^{[7,8]}$ For this reason, in the present study it was aimed to examine the effects of adaptive motion on TF NiTi rotary files shaping ability in Sshaped artificial canals.

According to the results of present study, it was determined that the adaptive motion has no statistically effect on TF NiTi rotary files' shaping ability. Thus, the null hypothesis of present study was accepted. Similarly to the present study results, Karataş et al. ${ }^{[8]}$ examined the shaping abilities of TF NiTi files operated with different kinematics on the mesial canals of mandibular molar teeth by using computed micro tomography. Authors reported that different kinematics had no statistically significant effect on TF NiTi rotary files' shaping ability. Also authors stated that this result originated from the fact that different kinematics did not affect the cutting efficiencies of files. Corroborating the present findings, You et al. ${ }^{[16]}$ examined the shaping abilities of ProTaper Universal (Dentsply Maillefer) performing continuous rotation motion and Reciproc (VDW, Munich, Germany) performing reciprocal motion, and reported that there was not statistically significant difference between the files in terms of canal transportation.

The increase of NiTi files' flexibility was reported to ensure the protection of original form of canal during the root canal shaping. ${ }^{[10]} \mathrm{TF}$ NiTi files are manufactured us- 
ing R-phase, and thus TF NiTi files have a flexible structure. ${ }^{[17]}$ For this reason, the adaptive motion might not influence the shaping abilities TF NiTi files. Besides that, Pedullà et al. ${ }^{[7]}$ reported that adaptive motion decreased the amount of canal transportation that is made by Mtwo (VDW) and TF NiTi files. We believe that this difference from the present study was originated from the use of natural teeth and the difference between the examination methods.

According to the present results, it was determined that there was not statistically significant difference between the groups in terms of the canal width at 11 points of S-shaped artificial canals after the shaping procedure, except for the $3^{\text {rd }}$ and $6^{\text {th }}$ points. At $3^{\text {rd }}$ and $6^{\text {th }}$ points, TFA group was determined to create fewer enlargements in artificial canals. As a reason for this, we believe that the stress occurring on the files during shaping the curved portions of S-shaped artificial canals was decreased via adaptive motion and the files remained more at the center.

\section{Conclusion}

Within the limitation this in vitro study, there was no statistically significant effect of adaptive motion on TF NiTi files' shaping ability in simulated S-shaped canals.

\section{Acknowledgment}

The authors thank to Yavuz Kanar for his assist during the photography process.

Conflict of interest: None declared.

\section{References}

1. Schilder H. Cleaning and shaping the root canal. Dent Clin North Am 1974;18:269-96.

2. Thompson SA, Dummer PM. Shaping ability of ProFile.04 Taper Series 29 rotary nickel-titanium instruments in simulated root canals. Part 1. Int Endod J 1997;30:1-7.

3. Schäfer E, Lohmann D. Efficiency of rotary nickel-titanium FlexMaster instruments compared with stainless steel hand K-Flexofile--Part 1. Shaping ability in simulated curved canals. Int Endod J 2002;35:505-13. Crossre

4. Capar ID, Ertas H, Ok E, Arslan H, Ertas ET. Comparative study of different novel nickel-titanium rotary systems for root canal preparation in severely curved root canals. J Endod 2014;40:852-6. Crossre

5. Schäfer E, Florek H. Efficiency of rotary nickel-titanium $\mathrm{K} 3$ instruments compared with stainless steel hand $\mathrm{K}$ Flexofile. Part 1. Shaping ability in simulated curved canals. Int Endod J 2003;36:199-207.
6. Gambarini G, Testarelli L, De Luca M, Milana V, Plotino G, Grande NM, et al. The influence of three different instrumentation techniques on the incidence of postoperative pain after endodontic treatment. Ann Stomatol (Roma) 2013;4:152-5. Crossre

7. Pedullà E, Plotino G, Grande N, Avarotti G, Gambarini G, Rapisarda E, et al. Shaping ability of two nickel-titanium instruments activated by continuous rotation or adaptive motion: a micro-computed tomography study. Clin Oral Investig 2016;20:2227-33. Crosste

8. Karataş E, Arslan H, Kol E, Bayrakdar İŞ. Effect of movement kinematics on canal transportation: reciprocation with different angles, adaptive motion, and continuous rotation. Turk Endod J 2016;1:13-8. Crosste

9. Saleh AM, Gilani PV, Tavanafar S, Schäfer E. Shaping ability of 4 different single-file systems in simulated Sshaped canals. J Endod 2015;41:548-52. Crossree

10. Madureira RG, Navarro LF, Llena MC, Costa M. Shaping ability of nickel-titanium rotary instruments in simulated S-shaped root canals. Oral Surg Oral Med Oral Pathol Oral Radiol Endod 2010;109:136-44. Crossree

11. Berutti E, Cantatore G, Castellucci A, Chiandussi G, Pera F, Migliaretti G, et al. Use of nickel-titanium rotary PathFile to create the glide path: comparison with manual preflaring in simulated root canals. J Endod 2009;35:40812. Crossse

12. Ding-Ming H, Hong-xia L, Cheung GS-P, Lan Z, Hong $T$, Xue-dong $Z$. Study of the progressive changes in canal shape after using different instruments by hand in simulated S-shaped canals. J Endod 2007;33:986-9. Crossse

13. Bonaccorso A, Cantatore G, Condorelli GG, Schäfer E, Tripi TR. Shaping ability of four nickel-titanium rotary instruments in simulated S-shaped canals. J Endod 2009;35:883-6. Crossse

14. Bergmans L, Van Cleynenbreugel J, Beullens M, Wevers M, Van Meerbeek B, Lambrechts P. Progressive versus constant tapered shaft design using NiTi rotary instruments. Int Endod J 2003;36:288-95. Crossre

15. Gergi R, Osta N, Bourbouze G, Zgheib C, Arbab-Chirani R, Naaman A. Effects of three nickel titanium instrument systems on root canal geometry assessed by micro-computed tomography. Int Endod J 2015;48:162-70. Crossre

16. You SY, Kim HC, Bae KS, Baek SH, Kum KY, Lee W. Shaping ability of reciprocating motion in curved root canals: a comparative study with micro-computed tomography. J Endod 2011;37:1296-300. Crosste-

17. Gambarini G, Gerosa R, De Luca M, Garala M, Testarelli L. Mechanical properties of a new and improved nickeltitanium alloy for endodontic use: an evaluation of file flexibility. Oral Surg Oral Med Oral Pathol Oral Radiol Endod 2008;105:798-800. 